\title{
La géométrie plane dans
}

\section{Combray}

\author{
Sandra Beyer
}

La création littéraire du village de Combray étant surtout une synthèse des impressions sensuelles du narrateur, 1'abondance d'images visuelles dans l'oeuvre frappe le lecteur tout de suite. Pour créer ces images, Proust se sert d'objets et de leur rapport spatial, de couleurs, de mouvements et de formes. Si on dessinait les images du roman, on aurait affaire quelquefois à une composition riche, d'autrefois délicate, et parfois ce serait une série de configurations surimposées les unes sur les autres. Ailleurs 1'auteur crée une image en trompe-1'oeil, où le lecteur ne voit que la fusion des objets ou des images. Avant de les dessiner, le lecteur ne saurait où mettre la fin d'un objet et le commencement d'un autre. Certes, on a longuement parlé de l'image impressioniste de Proust; et pourtant $1^{\prime}$ aspect analytique de ces images semble avoir été négligé par les commentateurs de son roman. A côté de la finesse de l'esprit de Proust, le côté analytique $n^{\prime}$ en est pas pour autant moins important.

Les formes géométriques se trouvent en abondance dans les "dessins visuels" de Proust. I1 trace souvent ces configurations géométriques au moyen de 1 'emplacement des objets dans 1 'espace. A cause du nombre de configurations dans ces passages descriptifs et à cause de la complexité des passages où il s'agit de la géométrie tridimensionnelle dont une discussion même sommaire ne nous sera pas possible dans les limites de cet article, nous nous bornerons par conséquent au vocabulaire spécifique de la 
géométrie plane qui se trouve dans Combray.

Les caractéristiques de la géométrie conviennent bien à 1'oeuvre de Proust. Dans la géométrie, il s'agit du rapport spatial et temporel des objets. La géométrie est 1'abstraction pure de la forme qui est gouvernée par des règles relatives qui peuvent changer. Les déclarations universelles n'ont pas de place dans la géométrie. La géométrie est un exercice de logique, mais la logique et la réalité ne sont pas toujours la même chose.

Toutes les formes de la géométrie plane se composent de lignes. La ligne, élément à la fois nécessaire, mais illusoire, est le terme géométrique dont Proust se sert le plus souvent. Quoique sur la page la ligne délimite toute forme géométrique, selon les mathématiciens, cette ligne n'existe pas. Pour ceux-ci, la ligne n'est qu'une série infinie de points, un point ayant une position mais pas de dimension. Un point est donc invisible. Nous pouvons représenter un point par une tache d'encre sur une feuille de papier et nous pouvons dessiner une ligne, mais ce ne sont que des représentations concrètes et inexactes de la forme dont il s'agit. La fonction géométrique d'une ligne est de diviser un plan.

C'est en parlant de "la 1igne" des clochers de Combray et de Martinville que Proust donne au mot le plus grand nombre de significations. Il commence par une étude de la perspective vis-à-vis des clochers. I1 détermine la géographie de son village et des environs par la position du clocher de l'église. Dans ce passage, la juxtaposition du clocher et des activités banales du narrateur (chercher les lettres à la poste, demander des nouvelles de Mme Sazerat, aller à la gare) sert à mettre en valeur le contraste entre la grandeur de cette structure et la vie humble et quotidienne du village. 
Les clochers de Martinville fournissent au narrateur plusieurs possibilités géométriques et nous constatons une progression dans le sens qu'il attribue au mot "1igne."

Au début, c'est le déplacement des lignes des clochers qu'il remarque, leur ensoleillement et leur déplacement semblent lui donner 1'occasion d'atteindre à un "moment sublime." Mais la combinaison de lignes et de la lumière, leur qualité irréelle et changeante 1'empêchent d'y atteindre mais en même temps l'attirent. Puis, le choix se présente. Il dit, " $\mathrm{j}$ 'avais envie de garder en réserve dans ma tête ces lignes remuantes au ciel et de n'y plus penser maintenant." (RTP, I, 180) Mais il y pense, faute de chose plus intéressante à faire et "Bientôt leurs lignes et leurs surfaces ensoleillées, comme si elles avaient été une sorte d'écorce, se déchirèrent, un peu de ce qui m'était caché en elles m'apparut et . . . pris d'une sorte d'ivresse, je ne pus plus penser à autre chose." (RTP, I, 180) Ce "moment sublime" mène à la création artistique, à une description poétique des clochers de Martinville. Quel rôle les lignes jouaient-elles dans cette création? Le jeu entre la ligne qui divise 1 'espace du ciel et les rayons du soleil produisent une réfraction de la lumière dans les yeux du narrateur. Cette lumière, que les savants représentent par une série de lignes, communique directement au "cerveau" ou au "centre créateur" du narrateur. Les lignes sont en quelque sorte des impulsions créatrices qui se "déchirèrent" dans son esprit et d'où il résulte une concrétisation de ce lien entre la perception visuelle et 1'esprit. Après ce "moment privilégié," les clochers ressemblent à "trois fleurs peintes sur le ciel au-dessus de la ligne basse des champs." (RTP, I, 182) L'interréaction complexe des lignes, des clochers, des rayons du soleil, et de 1 'oeil étant terminee, l'image devient celle d'un dessin d'enfant, structurellement simplifiée et dépourvue de signification. 
Proust ne se sert du mot "point" dans le sens géométrique que deux fois dans Combray. Un point n'a pas de dimensions, il n'a qu'une position, arbitraire d'ailleurs, car c'est le géomètre qui arrange les points où bon lui semble. Voyons où Proust, géomètre dans le sens plein du terme, met un point et ce qu'il veut dire par le mot: "Un homme qui dort tient en cercle autour de lui le fil des heures, 1 'ordre des années et des mondes. I1 les consulte d'instinct en s'éveillant et y lit en une seconde le point de la terre qu'il occupe. . ." (RTP, I, 5) Le narrateur continue en disant que cet ordre peut avoir changé avant notre réveil. Nous assignons à notre position un point, et nous le gardons constant, mais ce $n^{\prime}$ est que ce point dont nous contrôlons la position. L'ordre des choses (du monde) ou l'ordre du temps est indépendant de nous et de notre point arbitraire, qui n'a plus de réalité puisque $I^{\prime}$ environnement a tout à fait changé. Le changement de l'ordre objectif ou temporel autour de nous fait de notre effort, pour fixer le point que nous occupons, un exercice inutile. C'est en employant le point que Proust nous démontre la séparation de 1 'homme du temps et de I'espace.

Plus tard, le narrateur dit que sa chambre devient "le point fixe et douleureux" de ses préoccupations. (RTP, I, 9) Ses préoccupations existaient toujours, mais en leur donnant un "point fixe," il les représente par un symbole, sa chambre. Ce point arbitraire dans le roman, comme dans la géométrie, sert de point de départ à la pensée. Il nous donne une concentration, artificielle, mais au moins ouvrable, d'un problème si abstrait qu'on aurait des difficultés énormes à en parler sans l'aide d'une représentation quelconque.

L'angle, quoiqu'un élément important dans la géométrie dont les possibilités littéraires semblent diverses, fonctionne dans Combray d'une façon plutôt constante: C'est une forme négative 
représentant la froideur, la peine, et $1^{\prime}$ interruption du bien-être et de I'habitude.

En parlant d'une chambre hostile à Balbec, le narrateur décrit une "étrange et impitoyable glace à pieds quadrangulaire, barrant obliquement un des angles de la pièce." (RTP, I, 8) Toute la pièce semble être indifférente ou hostile envers lui, mais cette collection d'angles au coin devient le centre de la malveillance. Les angles deviennent des épines qui piquent 1 'habitude, et le miroir est là pour attirer son attention au coin par la semblance de mouvement et la lumière qu'elle transmet dans 1a pièce.

Le meilleur exemple de l'emploi de l'angle comme obstacle ou comme difficulté se trouve au moment où le père du narrateur suggère une promenade du côté de Guermantes. "On partait tout de suite après déjeuner par la petite porte du jardin et on tombait dans la rue des Perchamps, étroite et formant un angle aigu. . ." (RTP, I, 165) $D$ 'habitude, les rues sont droites ou courbées et leurs angles sont plus ouverts qu'un angle aigu, mais en se servant du mot "étroite" et de l'angle aigu, le narrateur met en valeur I'inaccessibilité de Guermantes pour lui.

Le cercle est une surface limiteé par une ligne courbe dont tous les points sont à égale distance du centre. C'est une forme structurellement forte parce qu'elle est repliée sur elle-même.

Le cercle est une surface qui donne au narrateur le sentiment de la sécurité. "Un homme qui dort tient en cercle autour de lui le fil des heures, 1 'ordre des années et des mondes." (RTP, I, 5) La ligne qui ferme le cercle est faite des heures, de I'ordre des années et des mondes. C'est une ligne dont le mouvement s'arrête pendant le sommeil du narrateur. Le narrateur se croit en sûreté parce qu'il peut s'endormir en se fiant à ce que $l^{\prime}$ ordre 
du temps ne va pas changer. Il se trompe, mais le mot "cercle" imp'lique ici la constance et la sûreté.

Proust décrit Combray en disant que les remparts du moyen âge cernaient la ville "d'un trait aussi parfaitement circulaire qu'une petite ville dans un tableau de primitif." (RTP, I, 48) Dans le texte, Proust nous trace une série de cercles concentriques: Le cercle religieux, le cercle pastoral, le cercle protégé par les remparts et le cercle de la France médiévale entourent le narrateur qui est au centre. Ce réseau de cercles protège le narrateur contre le monde extérieur, car une fois descendu du train, il se sent calme et loin de la vie extérieure. Mais les cercles servent aussi à limiter son monde pendant ses séjours à Combray. Il entre immédiatement dans " 1 'habitude" de Combray et dans le monde de sa tante et de Françoise. Il ne pense plus à la vie parisienne.

La ligne, le point, les angles, toutes les formes géométriques qui se trouvent dans Combray contribuent à renforcer l'aspect visuel aussi bien qu'à illustrer certains principes d'ordre intellectuel. Dans ce roman, le jeune narrateur se sert de toutes ses connaissances pour reconstruire le village qui aura une importance capitale dans le développement des grands thèmes d'A 1 a Recherche du temps perdu. La géométrie plane que le jeune écolier a certainement étudiée au lycée sera infiniment précieuse lorsque le narrateur mûri se mettra à traduire ses impressions, ses expériences et ses concepts en littérature.

Toutes les citations viennent de:

Proust, Marcel. A 1a Recherche du temps perdu, Vol. I. Paris: Gallimard (Bibliothèque de la P1éiade), 1954. 